\title{
Calculation of the Fine-Structure Constant
}

\author{
Jesús Sánchez \\ Independent Researcher, Bilbao, Spain \\ Email: jesus.sanchez.bilbao@gmail.com
}

How to cite this paper: Sánchez, J. (2018) Calculation of the Fine-Structure Constant. Journal of High Energy Physics, Gravitation and Cosmology, 4, 510-518.

https://doi.org/10.4236/jhepgc.2018.43029

Received: April 21, 2018

Accepted: July 3, 2018

Published: July 6, 2018

Copyright $\odot 2018$ by author and Scientific Research Publishing Inc. This work is licensed under the Creative Commons Attribution International License (CC BY 4.0).

http://creativecommons.org/licenses/by/4.0/

\section{Abstract}

The fine-structure constant $\alpha$ [1] is a constant in physics that plays a fundamental role in the electromagnetic interaction. It is a dimensionless constant, defined as:

$$
\alpha=\frac{q^{2}}{2 \varepsilon_{0} h c}=0.0072973525664
$$

being $q$ the elementary charge, $\varepsilon_{0}$ the vacuum permittivity, $h$ the Planck constant and $c$ the speed of light in vacuum. The value shown in (1) is according CODATA 2014 [2].

In this paper, it will be explained that the fine-structure constant is one of the roots of the following equation:

$$
\cos \left(\alpha^{-1}\right)=e^{-1}
$$

being $e$ the mathematical constant $e$ (the base of the natural logarithm). One of the solutions of this equation is:

$$
\alpha=0.0072973520977
$$

This means that it is equal to the CODATA value in nine decimal digits (or the seven most significant ones if you prefer). And therefore, the difference between both values is:

$$
\text { Difference }=\frac{\alpha(1)-\alpha(3)}{\alpha(1)} \cdot 100=0.00000642 \%
$$

This coincidence is higher in orders of magnitude than the commonly accepted necessary to validate a theory towards experimentation.

As the cosine function is periodical, the Equation (2) has infinite roots and could seem the coincidence is just by chance. But as it will be shown in the paper, the separation among the different solutions is sufficiently high to disregard this possibility.

It will also be shown that another elegant way to show Equation (2) is the following (being $i$ the imaginary unit): 


$$
e^{\frac{i}{\alpha}}-e^{-1}=-e^{-\frac{i}{\alpha}}+e^{-1}
$$

having of course the same root (3). The possible meaning of this other representation (5) will be explained.

\section{Keywords}

Fine-Structure Constant, Electromagnetism, CODATA Values, Atom, Electron, Quantum Numbers, Trigonometric Functions, Exponential Function

\section{Introduction}

The fine-structure constant $\alpha$ [1] plays a substantial role in the electromagnetic interaction and it is defined as:

$$
\alpha=\frac{q^{2}}{2 \varepsilon_{0} h c}=0.0072973525664
$$

being $q$ the elementary charge, $\varepsilon_{0}$ the vacuum permittivity, $h$ the Planck constant and $c$ the speed of light in vacuum. The value shown in (1) is according to CODATA 2014 [2].

In this paper, it will be shown that $\alpha$ is one of the roots of the following equation (being $e$ the mathematical constant $e$, base of the natural logarithm):

$$
\cos \left(\alpha^{-1}\right)=e^{-1}
$$

with the stunning precision of $0.00000642 \%$.

\section{Roots of the Equation}

We can solve Equation (2) using the following steps:

$$
\cos \left(\alpha^{-1}\right)=e^{-1}
$$

We apply the function arccosine to both sides of the equation:

$$
\begin{gathered}
\arccos \left(\cos \left(\alpha^{-1}\right)\right)=\arccos \left(e^{-1}\right) \\
\alpha^{-1}=\arccos \left(e^{-1}\right) \\
\alpha=\frac{1}{\arccos \left(e^{-1}\right)}
\end{gathered}
$$

Now, we have to be careful with the arccosine function. It is multivalued. Normally it is defined to take only the value between 0 and $\pi$. And all the results are separated from this value by multiples of $2 \pi$. This means the way of expressing Equation (7) having all the possible solutions is:

$$
\alpha=\frac{1}{\arccos \left(e^{-1}\right)+2 n \pi}
$$


Being $\mathrm{n}$ all the integer numbers. We can check that for $n=-22$, we have:

$$
\alpha=\frac{1}{1.19406881873632-22 \times 2 \pi}=-0.00729735209773166
$$

It could be surprising that the value is negative but this is not strictly true. It is negative due to the interval we have defined for the arccosine (the standard one between 0 and $\pi$ ). We can choose the interval between $-\pi$ and 0 , instead. As the cosine is an even function, the value of the arccosine in this range ( $-\pi$ and 0$)$, will be the same as in the standard interval (between 0 and $\pi$ ) but with opposite sign. This means that the value $-1.1940688 \ldots$ is also a valid value of the arc$\cos \left(e^{-1}\right)$. This is, the following equation-in which the arccosine is defined again according the standard interval $(0$ to $\pi)$-is also valid:

$$
\alpha=\frac{1}{-\arccos \left(e^{-1}\right)+2 n \pi}
$$

This time, we take $n=22$ to get:

$$
\alpha=\frac{1}{-1.19406881873632+22 \times 2 \pi}=0.00729735209773166
$$

And we get the positive value, that it is also a valid solution for Equation (2) as it can be checked easily:

$$
\begin{gathered}
\cos \left(\alpha^{-1}\right)=e^{-1} \\
\cos \left(0.0072973520977316^{-1}\right)=0.3678794411714 \\
e^{-1}=2.71828182845905^{-1}=0.3678794411714
\end{gathered}
$$

But one question we can have now is the following. As the Equation (2) has infinite solutions, could it be that they are so many, that one of them will have in any case the value we want? The comparison with the real value of the fine structure constant will be checked in the next chapter. But what we can do now is to look for the neighbor solutions of the ones we have taken. Are they very near? Or is there enough separation to start disregarding the problem "I have chosen the solution I want" issue?

The nearest solutions to the value calculated in (11) are:

$$
\begin{aligned}
& \text { In Equation (10) with } n=22 \text { we find } 0.00717235881822185 \\
& \text { In Equation (10) with } n=23 \text { we find } 0.00751083661989347
\end{aligned}
$$

The comparison with the real value of the fine-structure constant will be done in the next chapter, but here we can at least calculate the relative distance between solutions. Are they sufficiently near to say that we are cheating choosing the solution we need from an infinity variety of solutions?

$$
\begin{aligned}
& \text { Distance }=\frac{(11)-(14)}{(11)} \cdot 100=\frac{0.0072973 \cdots-0.0071723}{0.0072973} \cdot 100=1.71 \% \\
& \text { Distance }=\frac{(15)-(11)}{(11)} \cdot 100=\frac{0.0075108 \cdots-0.0072973}{0.0072973} \cdot 100=2.92 \%
\end{aligned}
$$


These distances in the order of units of percentage could seem small. But as we will see in the next chapter 3, the degree of precision between value in (11) compared to measured value of the fine-structure constant is so high, that we will see that these distances are really huge. The distances are sufficiently high to say that the coincidence between values is not related to having a lot of solutions and choosing the one that it is nearest of the value we need.

So, as a summary of this chapter, we can say that we have calculated in (11) that the value 0.00729735209773166 is a root of the following Equation (2):

$$
\cos \left(\alpha^{-1}\right)=e^{-1}
$$

We have calculated also the neighbor solutions to this one. With this information, we will go the next chapter. We will compare this value with the measured value of the fine-structure constant.

\section{Comparison of the Root of the Equation with the Measured Value of the Fine Structure-Constant}

We have calculated that the roots of the equation:

$$
\cos \left(\alpha^{-1}\right)=e^{-1}
$$

can be found using the following (being $n$ an integer):

$$
\alpha=\frac{1}{-\arccos \left(e^{-1}\right)+2 n \pi}
$$

For the specific case when $n=22$, we get the root:

$$
\alpha=\frac{1}{-1.19406881873632+22 \times 2 \pi}=0.00729735209773166
$$

In parallel we have that the CODATA 2014 [2] value of the fine-structure constant is:

$$
\alpha=0.0072973525664
$$

The first comment we have, just comparing them, is that they are exactly the same in the first nine decimal digits (0.007297352) (seven if we only count significant digits).

If we calculate the difference in percentage we have:

$$
\text { Difference }=\frac{\alpha(11)-\alpha(18)}{\alpha(11)} \cdot 100=0.00000642 \%
$$

This precision in coincidence is orders of magnitude higher that the exactitude needed in an experimental result to validate a theory. If we see it in parts per unit, it corresponds to a coincidence up to $6.42 \times 10^{-8}$ parts per unit. Or you can see it as 0.0642 parts per million.

Now, compare this with the $1.71 \%$ and $2.92 \%$ distance to the neighbor solutions in (16) and (17). The distance is so high compared to the precision in coincidence that I do not know really what else to comment here. 


\section{Exponential Form of the Equation}

We can transform the Equation (2) to an exponential form doing the following steps.

The cosine function has the following exponential form as it can be checked in [3], being $i$ the imaginary function:

$$
\cos (x)=\frac{e^{i x}+e^{-i x}}{2}
$$

Now, we apply above Equation to (2):

$$
\begin{gathered}
\cos \left(\alpha^{-1}\right)=e^{-1} \\
\cos \left(\alpha^{-1}\right)=\frac{e^{\frac{i}{\alpha}}+e^{-\frac{i}{\alpha}}}{2}=e^{-1} \\
e^{\frac{i}{\alpha}}+e^{-\frac{i}{\alpha}}=2 e^{-1} \\
e^{\frac{i}{\alpha}}+e^{-\frac{i}{\alpha}}=e^{-1}+e^{-1} \\
e^{\frac{i}{\alpha}}-e^{-1}=-e^{-\frac{i}{\alpha}}+e^{-1}
\end{gathered}
$$

where Equation (21) is the same as Equation (2) but expressed via exponential functions. This means, the root 0.00729735209773166 keeps being a root also for Equation (21).

The first thing that draws attention of Equation (21) is the beauty of its antisymmetry. All the signs are interchanged between left and right-hand side except the last exponential $e^{-1}$ that should be $e^{+1}$. This breaks the symmetry of the antisymmetry whatever this means.

I will explain the few things that we can conclude regarding this equation in Chapter 5.3.

\section{Interpretation of the Equation}

As you can imagine, if I knew it, that would be one of the first things I would have explained in the paper. At this stage, is unknown. We only know that it works. Anyhow, there is some information we can get from it.

\subsection{Interpretation of the Cosine}

We will start with the interpretation of the $\cos \left(\alpha^{-1}\right)$ in the equation.

Let's not forget the origin of the fine-structure constant. It is the relation of the speed of the electron compared to the speed of light, in the first level of an atom [4]. It is also related to the relation of the Bohr radius of an atom to the Compton wavelength of an electron [4]. This means, we could try to relate it the electromagnetic interactions in the atom.

Let's see the Equation (2) once again:

$$
\cos \left(\alpha^{-1}\right)=e^{-1}
$$


If we check the equation literally, the left-hand side is a cosine, so we could understand that $\alpha^{-1}$ is an angle in radians. If we take $\alpha(11)$ The value of $\alpha^{-1}$ is:

$$
\alpha^{-1}=0.00729735209773166^{-1}=137.036007939215
$$

As one turn is $2 \pi$, this is higher than one turn. Let's separate in turns and check the remaining angle:

$$
\alpha^{-1}=137.036007939215=21 \times 2 \pi+5.08911648844324
$$

This means, this angle corresponds to 21 turns and a remnant angle 5.0891 ...radians. It also can be seen as:

$$
\alpha^{-1}=137.036007939215=22 \times 2 \pi-1.19406881873633
$$

That would mean almost 22 turns, not arriving by this $-1.1940 \ldots$ radians. This is logic as it is the way $\alpha$ was defined in Equation (11).

Now that we understand $\alpha^{-1}$ as an angle, let's go back to the atom. Imagine that the nucleus emits a photon that has an intrinsic property related to the electromagnetic interaction that is represented by a vector. This vector can rotate as the photon moves along or could be static (we do not know).

In parallel, the electron has a complementary property related to the electromagnetic interaction also represented by a vector (that also can be rotating or static).

So, when the photon arrives to the electron, the electromagnetic interaction between them is related to relative positioning of these vectors at the moment of interaction. When we talk about relative positioning between vectors, we can talk about projection of one of them onto the other. Or even we can talk about the most common scalar or dot product (that takes into account the angle between the vectors to obtain the result of the product) [5]. Anyhow, in both cases, this relation between the position of the vectors is shown using the cosine of the angle between them [5]. This means, in Equation (2) the $\cos \left(\alpha^{-1}\right)$ would be related with the interaction of these two properties of the photon and of the electron. More accurate, it would be related to the relative positioning of their vector (or in general orientation) at the moment of interaction.

Even, we could think about, in possibilities like when this angle of interaction is not $\alpha^{-1}$ or in the range of it, the interaction is too small or even inexistent. This would explain that only certain levels of energies of the electron exist in the atom. In the positions where for whatever reason this interaction does not have a certain angle (due to distances, synchronizations, rotations etc...) the electron does not interact with the photon. And it tends to go to stable levels where the photons interact and tends to keep the electron in that orbit.

One logic question we could have is: and why $\alpha^{-1}$ is higher than $2 \pi$ ? The cosine will be the same in a lower value than $2 \pi$. And even, if we take the above model literally, the angle between vectors will be always lower than $2 \pi$. The only explanation I can think about is that the Equation (2) is not an equation of a theory, but a remnant (a sub product if you want) of a more complex equation, after several simplifications or stablishing specific points of operation (boundary 
conditions). This means, $a^{-1}$ is not only the final angle of interaction, but includes also, for example the number of rotations that the vector of the photon or of the electron has made before the interactions. Probably, to arrive to this stability point where the electron has an allowed orbit, some synchronization has to be done between the number of rotations of the electron vector, the time to the photon to arrive etc... that gives that these 21 rotations (see Equation (23)) are necessary to arrive to the remnant angle $5.0891 \ldots$

\subsection{Interpretation of the Right-Hand Side of the Equation}

Finding an interpretation for this is really difficult, so this chapter will be even more speculative than the previous one. As the meaning of $e^{-1}$ is really obscure, what we can do is, instead of trying to understand it, we will try to make reverse engineering of which type of equations could lead to that result.

First, we have the obvious:

$$
\int_{-\infty}^{-1} e^{x}=e^{-1}-e^{-\infty}=e^{-1}
$$

But it is more interesting, the following:

$$
\int_{1}^{\infty} e^{-x}=-e^{-\infty}+e^{-1}=e^{-1}
$$

A typical meaning of an equation like this would be the following: $e^{-x}$ would represent a field or a magnitude (with spherical symmetry and that is zero at infinity) that has to be integrated from a certain point towards infinity. This "certain point" could be the Bohr radius, the radius of the electron or the radius of the proton or something related. The issue is that this point or radius is not given in meters, but in normalized units. This means, the variable $\mathrm{x}$ would be:

$$
x=\frac{r}{r_{\text {origin }}}
$$

So, the starting point would be 1 . And that is the reason that the value of the magnitude $e^{-x}$ once integrated is $e^{-1}$. As you can see, all of this, is completely speculative and just some ideas of where the Equation (2) could come from. And as commented in 5.1, all points to the idea that Equation (2) is not a complete equation of a theory but the result of a more complex equation, when applied at a specific point or boundary conditions.

Another typical way of getting $e^{-1}$ would be in an equation like the following:

$$
-1=\int_{a}^{b} \frac{f^{\prime}(x)}{f(x)}=[\ln (f(x))]_{a}^{b}=\ln \frac{f(b)}{f(a)}
$$

Now if we apply the exponential to both sides, we get:

$$
e^{-1}=\frac{f(b)}{f(a)}
$$

In the case of Equation (2), we even could try to have an idea of what $f(x)$ could be, as it should hold: 


$$
e^{-1}=\frac{f(b)}{f(a)}=\cos \left(\alpha^{-1}\right)
$$

This means, that the $\cos \left(\alpha^{-1}\right)$ should be the result of the division of the value of a function in two different points. The different possibilities could be checked via trigonometric relations or even the exponential representation. And probably we could try to understand why the Equation (28) was defined in the first place.

\subsection{Interpretation of the Exponential Form of the Equation}

In chapter 4, we found the following exponential form of the equation:

$$
e^{\frac{i}{\alpha}}-e^{-1}=-e^{-\frac{i}{\alpha}}+e^{-1}
$$

And it was demonstrated that was exactly the same equation as (2) with the same roots.

As we know, the exponentials that only have imaginary part (not real part in the exponential) represent rotations in the complex plane [3]:

$$
e^{\frac{i}{\alpha}}=\cos \left(\alpha^{-1}\right)+i \sin \left(\alpha^{-1}\right)
$$

This is in line of what we have commented in chapter 5.1. If we understand that this is related to some kind of interaction between vectors of the photon and the electron, these exponentials would represent the orientation or the rotation of them at that moment. And the equation would mean a constraint or restriction that should apply, for the interaction to occur (or occur with certain values).

So this would explain (if we can use that word) somehow the elements $e^{1 / \alpha}$ and $e^{-1 / \alpha}$. But the meaning of the elements $e^{-1}$ keep completely unknown apart for the possible explanations already commented in Chapter 5.2.

Another thing we can comment is that we can get rid of these $e^{-1}$ just multiplying by $e$ both sides of Equation (21):

$$
\begin{gathered}
e \cdot e^{\frac{i}{\alpha}}-e \cdot e^{-1}=-e \cdot e^{-\frac{i}{\alpha}}+e \cdot e^{-1} \\
e^{1+\frac{i}{\alpha}}-1=-e^{1-\frac{i}{\alpha}}+1
\end{gathered}
$$

Just in case the Equation (32) is in a form that could have a meaning in any discipline of mathematics or physics I am not aware of.

\section{Conclusions}

In this paper, we have explained that the fine-structure constant has this measured value according to CODATA 2014 [2]:

$$
\alpha=\frac{q^{2}}{2 \varepsilon_{0} h c}=0.0072973525664
$$

And we have shown that the following equation:

$$
\cos \left(\alpha^{-1}\right)=e^{-1}
$$

has a root that is 


$$
\alpha=0.0072973520977
$$

This means, it is equal in the 9 first decimal digits and has a difference compared to the measured value of:

$$
\text { Difference }=\frac{\alpha(1)-\alpha(3)}{\alpha(1)} \cdot 100=0.00000642 \%
$$

That is much higher in coincidence than the commonly accepted necessary to validate a theory towards experimentation.

Later some interpretations of Equation (2) have been given, mainly understanding that it should be related with the electromagnetic interactions inside the atom. Also, the following exponential form of Equation (2) has been commented:

$$
e^{\frac{i}{\alpha}}-e^{-1}=-e^{-\frac{i}{\alpha}}+e^{-1}
$$

\section{Acknowledgements}

To my family, friends, work colleagues, university colleagues, Task Gravitation team, and physics group. To all the things that make this world worth of it: to Fortaleza de Manpang webpage, to the Longest Journey, to Tea Abrana, to the most piraaata! fan, to my mom, to the one that invented the music videos, to the Best Film Ever by Zack/Deborah Snyder and Steve Shibuya on 2011 and to the I got a feeling of the Black Eyed Peas.

\section{References}

[1] Bouchendira, R., Cladé, P., Guellati-Khélifa, S., Nez, F. and Biraben, F. (2010) New Determination of the Fine-Structure Constant and Test of the Quantum Electrodynamics. Physical Review Letters, 106, Article ID: 080801.

[2] Mohr, P.J., Taylor, B.N. and Newell, D.B. (2015) The 2014 CODATA Recommended Values of the Fundamental Physical Constants (Web Version 7.0). http://physics.nist.gov/constants.

[3] Euler, L. (1748) Chapter 8: On Transcending Quantities Arising from the Circle of Introduction to the Analysis of the Infinite, 214, Section 138. Bruce, I., Trans. http://www.17centurymaths.com/contents/euler/introductiontoanalysisvolone/ch8v ol1.pdf

[4] Orchin, M., Macomber, R.S., Pinhas, A. and Wilson, R.M. (2005) Atomic Orbital Theory. http://educypedia.karadimov.info/library/0471680281.pdf

[5] Lipschutz, S. and Lipson, M. (2009) Linear Algebra (Schaum's Outlines). 4th Edition, McGraw Hill, New York. 